\title{
Prognostics and health management technology for radar system
}

\author{
Yongle Lyu ${ }^{1, *}$, Zhuo Pang ${ }^{1}$, Chuang Zhou ${ }^{1}$, and Peng Zhao ${ }^{1}$ \\ ${ }^{1}$ Nanjing Research Institute Of Electronics Technology, Jiangsu, China
}

Keywords: Radar system, Prognostics, Health management, Built-in test.

\begin{abstract}
Information-based war in the future has a higher requirement to the maintenance and support ability of radar system. Prognostics and Health Management(PHM) technology represents the research hotspot of maintenance system, and following key techniques need to be resolved to research on the radar PHM technology such as the acquirement and selection of health information and fault signs of a radar's electronical components, mass data warehousing and mining, fusion of multi-source test data and multi-field characteristic information, failure model building and forecasting, automatic decision-making on maintenance, and at the same time improving the self built-in test abilities of radar's components based on the optimization of Design For Testability(DFT). The radar PHM technology has the trend of "built-in to integrate", "together with DFT" and "long-distance and distributed". However, subjected to radar's complexity and current PHM technique level, radar PHM engineering still meets many challenges, but has bright future.
\end{abstract}

\section{Introduction}

Radar is a complex electronic information system which plays an important role in national defense and modernization. In recent years, with advancement of the related technology, the higher levels of digitization, integration and intelligence have been achieved, which is with increased difficulty of comprehensive maintenance operations. After being put into service, radar's guarantee capability has become a vital indicator of its performance. In future information war, it is urgent to explore a new security system that include aspects such as design, develop, produce, employ and maintain in order to adapt to the higher requirements of the rapid response capability, fault isolation capability and wartime repair capability. This system also coordinates resources and quickly completes the comprehensive support tasks of the new radar system.

Since the 1990s, with the development and wide application of information technology, the construction of comprehensive maintenance operations becomes the integration of testing, monitoring, diagnosis, forecasting and maintenance management, and finally produces fault prediction and health management techniques ( $\mathrm{PHM}$ ). PHM technology is a

\footnotetext{
*Corresponding author: nriet306_lyl@,126.com
} 
further extension of the state monitoring function, with a distinctive feature of the introduction of predictive capabilities [1].

PHM technology takes the current state of the equipment as the start point, combined with the structural characteristics, parameters, environmental conditions and historical data of the object, to monitor the current working state of the equipment by various knowledge, algorithms and models based on advanced sensor acquisition technology. Then fault of the equipment are predicted, analyzed, judged and its type, extent, cause and location are determined. PHM technology calculates the residual using life of the equipment and formulates a scientific maintenance plan based on the acquired information, intending to eliminate faults in advance to ensure the completion of training and combat missions [1-3].

PHM technology represents the trend in the maintenance mode, which replaces traditional break maintenance or periodical inspection with condition-based maintenance. Implementing the PHM project on the radar not only can timely inform people of the health status and development trend of the radar, ensure its integrity of system, operational safety and mission reliability, but also can reduce the scale of protection, maintenance costs, shorten maintenance time, and improve the comprehensive support capability of the radar.

The electronic components have the largest proportion and the most complicated structure in the radar system. Whether the electronic components work steadily or reliably directly affect the mission success rate of the radar system. From the viewpoint of economic benefits, the cost of the design, development, manufacture and maintenance of the electronic components in the radar system account for the highest proportion. Therefore, the electronic components are the hotspot of the PHM research of the radar system. In addition, radar subsystems, such as servo subsystem, also contain mechanical components. The theoretical method for fault prediction of mechanical components is relatively mature and thus not focused in this paper. Readers can refer to reference [1] for more details.

\section{Research status of PHM technology in electronic systems}

As early as July 2000, PHM technology, which is a comprehensive technology that significantly reduces costs of system usage and security and improves system security and availability, was included in the US Department of Defense's report "Key Technologies of Military" [4].

The research and application of PHM technology abroad indicates that PHM is becoming a key technology for the design of a new generation of large-scale complex equipment systems. The US Army's "Health \& Usage Monitoring System (HUMS)" integrated application platform is characterized by the functions of fault diagnosis, usage monitoring and cross-linking with the maintenance support system, which can be regarded as an early representative of the PHM system [5]. Currently, several types of helicopters including the AH-64 Apache and the UH-60 Black Hawk are equipped with the HUMS. The UK Ministry of Defense also reached an agreement with Smith Aerospace Company to develop a status monitoring system in combination with a cockpit voice/flight data recorder for future Bobcat helicopters, which was delivered in 2011. In addition to helicopters, PHM technology has also begun to be put into service on fixed wing aircrafts. F-22, Rafale fighter, B-2 bomber, C-130 Hercules transport aircraft, RQ-7A/B shadow 200 tactical unmanned aircraft set up a PHM system named Integrated Vehicle Health Management System (IVHMS), which has capabilities of online condition monitoring management, fault detection, fault prediction, fault isolation, system reconfiguration and information transfer to the ground support system [6-8]. Health management systems have been applied to the US X-Series supersonic aircrafts at the beginning of the design. The PHM technology employed by the Boeing 787 and the Joint Strike Fighter F-35 represents the highest level in the world [9]. 
It is difficult to predict the fault of the electronic system due to its physical characteristics. The US military has proposed an argument that the failure prediction of electronic system is not achievable. However, with the improvement of electronic system prediction technology, the US Department of Defense pointed out in 2004 that electronic system fault prediction is the critical technology to realize independent logistics support, and carried out many researches on electronic system fault prediction technology based on the JSF project [10]. In March 2006, the United States established the Electronic Forecasting and Health Management Association [11].

Currently, the University of Maryland's Center for Advanced Life Cycle Engineering (CALCE) represents the top level in the field of electronic PHM technology research [12].

\section{Key technology of radar PHM system}

\subsection{Health information and prediction parameter acquisition technology}

Generalized radar health information includes technical data, history record, as well as work and environmental stress levels, which is related to the design, use, maintenance and repair of radar systems, and helps to evaluate the mission capabilities, identify the damage residues and hidden dangers, and develop a scientific maintenance plan [11].

And narrow sense of radar health information refers to the parameters which can demonstrate the operating status and performance of radar systems and components, and mainly includes two categories: signal value and information value. Signal value contains electrical signals such as transmit and receive waveform parameters (cycle, pulse width, top drop, etc.), spectral characteristics (main to side ratio, bandwidth, etc.), excitation power, and so on, which obtained directly or after conditioning from BITE, PMA, and ATE. While non-electrical signals contains hydraulic, temperature, vacuum, optical signals and so on, and usually obtained by dedicated sensors. The information value mainly includes various performance indicators generated by the operations of data processing computer, such as main lobe width after pulse compression, ranging power, pattern parameters and so on, which is transmitted via radar intranet or various buses.

Prediction parameter is usually a measurable variable related to damage which can sensitively reflect the occurrence and development of potential failures. For example, the output voltage of the switching power supply is offset, which may imply the damage to the feedback regulator or isolator. And ripple voltage gradually increases, which may mean that the filter capacitor is aging.

When design a radar PHM system, prediction parameters are chosen by the physical model and failure mode of the detected object. And the commonly used prediction parameters of electronic devices are shown in Table 1 [12].

Table 1. Commonly-used prediction parameters of electronic devices in radar.

\begin{tabular}{|c|c|c|}
\hline No. & Electronic Devices & Prediction Parameters \\
\hline 1 & $\begin{array}{l}\text { Switching power } \\
\text { supply }\end{array}$ & $\begin{array}{l}\text { DC voltage and output current stability, ripple voltage, pulse width } \\
\text { ratio, feedback, leakage current, RF noise, temperature, etc. }\end{array}$ \\
\hline 2 & $\begin{array}{l}\text { Conductor and } \\
\text { connector }\end{array}$ & Loss, \\
\hline 3 & Capacitor & Leakage current, impedance, dissipation factor, RF noise, etc. \\
\hline 4 & Diode & Forward voltage drop, thermal resistance, power consumption, etc. \\
\hline 5 & Amplifier & Gain, frequency response. \\
\hline 6 & Photoelectric coupler & Forward voltage drop, current conversion ratio. \\
\hline 7 & RF power amplifier & Voltage standing-wave ratio, power consumption, leakage current. \\
\hline 8 & Time-based circuit & Output frequency, power loss, phase distortion, noise, etc. \\
\hline
\end{tabular}




\begin{tabular}{|l|l|l|}
\hline & $\begin{array}{l}\text { Voltage-controlled } \\
\text { oscillator }\end{array}$ & \\
\hline 9 & CPU & Bus operation, bus clock; Address signals and address latch signals. \\
\hline 10 & AD/DA & Conversion accuracy and linearity. \\
\hline 11 & Memory & Correctness of full address range data access. \\
\hline 12 & $\begin{array}{l}\text { Communication } \\
\text { Interface }\end{array}$ & $\begin{array}{l}\text { Output signal amplitude, transceiver function, communication } \\
\text { protocol, invalid data error bit rate. }\end{array}$ \\
\hline 13 & PLA & $\begin{array}{l}\text { Logic function, static current, current range, logic level, operating } \\
\text { features. }\end{array}$ \\
\hline
\end{tabular}

In Engineering applications, not only the level of fault monitoring ( unit, module, component, etc.), failure mode and its occurrence probability, severity, propagation, but also the difficulty and complexity of measurement points, the influence on weight, volume, power consumption, and electromagnetic environment of monitoring object should be taken into consideration when choosing proper prediction parameters of radar electronic components. Based on multi-signal model [13], the test design can use methods like rough set theory [14], neural network [15], and evolutionary computation [16] to mine the dependence in the prediction parameters and eliminate redundant points, which can also help to improve the utilization of prediction parameters, reduce unnecessary measurement points, and save costs of getting radar health information.

\subsection{Database building and data mining technology}

The PHM project for radar involves large amount of data and various categories, which are still growing as time goes by. The main content in database building and data mining include how to store, organize and manage these data effectively and improve the efficiency and utilization rate of data.

The built database should be able to store radar operation status, environmental stress, fault history, monitoring and diagnosis results, together with maintenance and repair records in chronological order. It should also store the setting data of PHM software when loading, running, data processing, displaying. Data and knowledge related to radar fault classification, diagnostic (such as normal tolerance, abnormal criteria, etc.), data related to composition, operation and maintenance mechanism, including instrument maintenance information, system log, operation tips and so on, are also necessary.

Data mining is the extraction of implicit and potentially-useful information from a database. For example, the deterioration trend and fault evolution rule of radar unit or component can be mined from the historical data of radar operation state. And fault criteria and diagnosis knowledge can be mined from a large number of historical fault data and repair records. Data mining generally requires data preprocessing, modeling and mining, interpretation and evaluation [17]. Commonly used data mining methods mainly includes inductive learning [18], cluster analysis [19], statistical modeling [20], evidence theory, Bayesian inference [21], and artificial neural network, etc.

\subsection{Information fusion technology}

Information fusion technology integrates as much information as possible through various intelligent algorithms to obtain comprehensive and more reliable evaluation results [22]. Information fusion can be divided into three levels: sensor layer (low information distortion, large amount of data), feature layer, and inference layer (integrating knowledge and experience). 
The information fusion of radar PHM system in sensor layer is mainly reflected in the data fusion among BIT, near-field PMA test, and far-field ATE test. The information fusion in feature layer is mainly manifested in the sign detection and recognition process, including obtaining fuller warning information by various detection methods from time, frequency, space, and data domain [23], and eliminating redundancy and interference by PCA and correlation analysis. In inference layer, the fusion is embedded in the joint use of design and manufacturing information, maintenance data, and fault diagnosis knowledge of radar unit components.

\subsection{Fault prediction technique}

The fault prediction of radar system can dynamically track and monitor the changes in the performance of radar unit components, identify and manage the occurrence and development of the fault, and provide sufficient time and decision basis for the fault prevention and clearance. It can also predict the future health of radar state, determine the residual life, plan maintenance and ensure supply [1].

According to the physical features of radar, the following methods can be adopted for fault prediction:

(1) Fault prediction based on reliability data

Based on the component failure model provided by the manufacturer, the fault model of radar components, units, subsystems, and even systems of level 1 can be established. The MTBP index is estimated by the reliability data accumulated during the use of radar, and then the estimated remaining life is equal to the MTBF minus the working time. This method can be used when similar electronic equipment is large and failure data is abundant. The disadvantage is that the significance of statistical indicators is not great specific to the one individual object, and it is difficult to determine the reliability index after the equipment repairs (especially after the replacement of components).

(2) Fault prediction based on prediction parameter monitoring

By FMECA analysis, physical parameters related to radar system failure or performance degradation are selected as prediction parameters for monitoring and a model can be established for fault prediction based large number of monitoring data. For the differences of radar function, structure, and failure mechanism, the selection of prediction parameters and data processing methods are also various. For example, ripple voltage can be used to monitor the health status of the high-voltage power supply in the radar transmitter, and cathode current can be used to monitor the klystron of the transmitter.

In engineering, multi-signal model can be used to establish the dependence between failure mode and monitoring parameters. The monitoring parameter with the highest correlation degree can be selected as the prediction parameter. And this method also has several following difficulties: a) Model selection problem: for a given fault mode, it is difficult to select the most consistent prediction model from numerous models. b) Data quality problem: prediction model parameters are estimated by historical data, and the error information or interference factors in data will greatly affect the model accuracy.

(3) Fault prediction based on cumulative damage model

The cumulative damage model [24] use the geometry, load state, and material features in the radar life cycle to analysis and identify the potential failure mechanism, and monitor the environment and stress in each fault part, then calculate cumulatively and infer the residual life of the product.

This method requires a thorough understanding of the root cause and formation process of the fault, and has the advantage of taking the environmental, mechanical (vibration, impact) factors into account. It can also be used to assess the damage in non-operation state.

(4) Fault prediction based on early warning circuit 
Early warning circuit is often used to predict the failure of semiconductor devices, and usually preset in the components. This technology has been put into practice in foreign countries, for example, Ridgetop. Group in USA has established a corresponding physical model and developed a fault warning circuit for electrostatic damage, electro-migration, thermal carriers, radiation damage, and other failure mechanisms, which can fail when the remaining $20 \%$ of the life of the host device [25].

\subsection{Maintenance decision technology}

Maintenance decision [26] is the ultimate goal of radar system PHM, that is, on the basis of the performance prediction analysis and health assessment of the radar, the maintenance support resources and technical forces are coordinated and rationally deployed, and the maintenance strategy is automatically generated.

In view of the uncertainty of forecasting and evaluation conclusions, it is necessary to comprehensively examine the severity of fault and performance degradation, design redundancy, test cost, maintenance cost factors, and conduct "risk-benefit" analysis to achieve maintenance decisions that tolerate uncertainty. There are two types of maintenance strategies: a) Isolate the parts that may cause the failure, and make changes in the system performance indication through measures such as replacement, compensation, and reconstruction. b) For the tolerable performance degradation, estimate the remaining life of the radar system to continue safe and stable operation, and guide the user to take measures for reasonable maintenance.

\section{BIT design for radar PHM}

In traditional maintenance systems, BITs (Built in Tests) are employed just for fault diagnostics of radar LRUs (Line Replaceable Units), the health information for PHM is very restricted. Usually only several characteristic signal or state parameters are collected, but without detailed diagnostic analysis or fault prediction. Application of radar PHM requires much more detailed and essential performance parameters and running status information of radar subsystems, units, components and even circuit boards. This needs improvement of traditional BITs based on radar testability design and optimization.

Figure 1 shows the upgraded BIT system, which consists of module BITs, subsystem BITs and central BITE (Built-in-Test Equipment). It supports both online monitoring and offline analysis of radar operating performances and running status. The central BITE, which consists of in-situ testing equipment and PHM software running on the terminal PC, as Figure 2 shows, integrates functions of state monitoring, auto-testing, fault diagnostics, fault prediction and health assessment. As a new measure of optimization for radar testability, the central BITE contributes significantly in monitoring and diagnosing the interface faults and link faults, moreover, the derived faults from fault propagation and coupling in radar. Consequently, the central BITE efficiently overcomes the defects in fault detection and isolation by regular module BITs and subsystem BITs. 


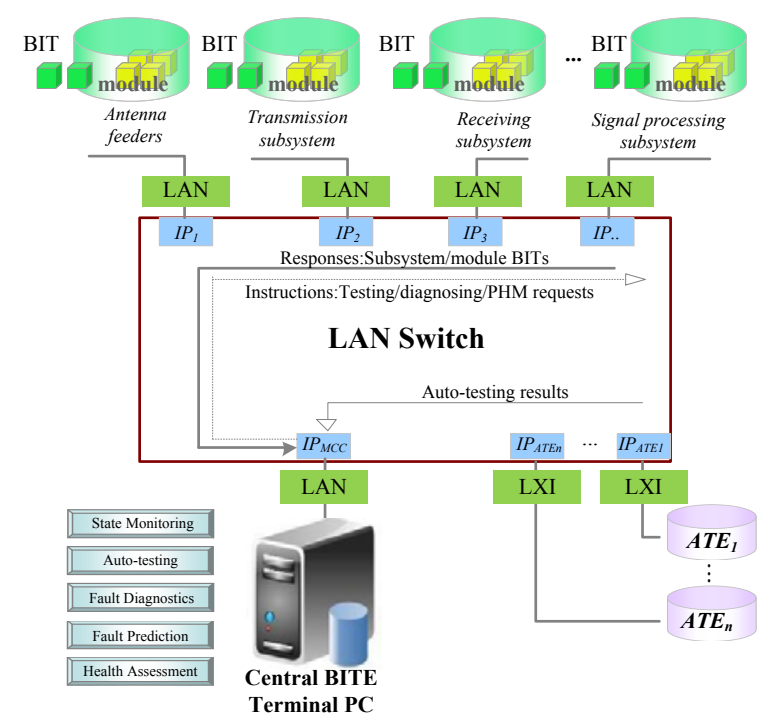

Fig. 1. Radar BITE for PHM.

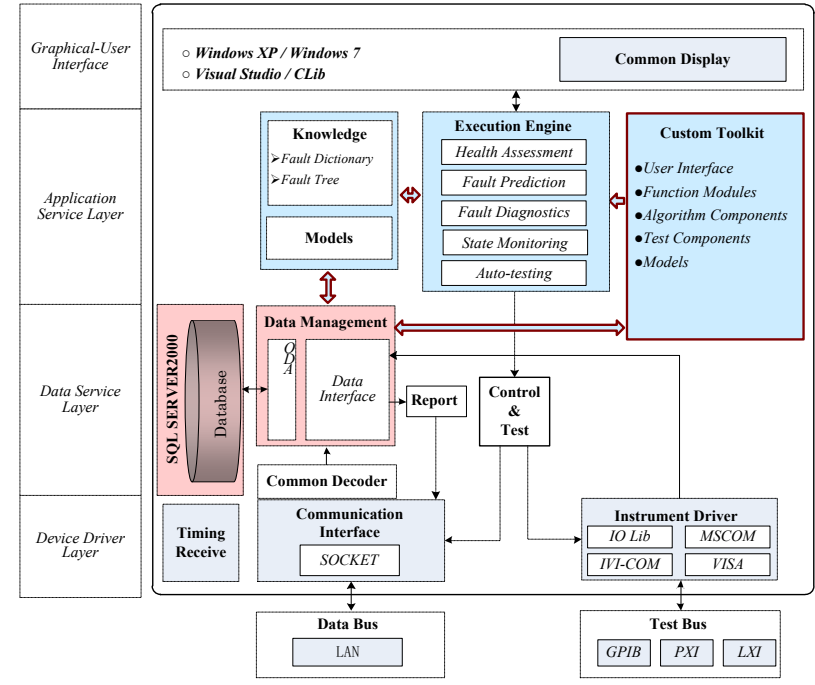

Fig. 2. Software architecture of radar PHM.

Online monitoring is conducted during the radar mission period and mainly used to:

(1). Collect and process the health condition parameters regularly;

(2). Monitor performance and function abnormalities inside the radar system, units and components;

(3). Find the fault warnings timely;

(4). Assess the risk level of potential faults.

Online monitoring must satisfy the requirement of real-time processing and response. Once a potential fault with high risk level is detected, the radar system should be informed immediately to save data and quit the mission execution. Differing from traditional post-fault diagnosis, early fault prediction and prevention is more of concern here for guarantee of the mission reliability. 
Offline analysis is conducted in radar debugging or maintenance state. It makes deep analysis on the fault warnings or potential faults with the help of in-situ test equipment and externally powerful computing resources, so as to determine the fault authenticity and possible fault location, as well provide as much guidance as possible for the follow-up workshop maintenance and repairing. On the premise of without increasing BITE scale and without affecting the real-time monitoring and processing, the offline analysis improves the test coverage, computing and information sharing ability of BIT. To achieve more accurate analysis, it is necessary to make full use of the BIST functionality of VLSI devices provided by the manufacturers, and embed self-checking program in the programmable logic devices within the module. In radar offline state, through test measures like bypass, loopback and boundary scan, the offline analysis achieves test of underlying functional circuits, integrity check of signal channels, and fault detection and isolation. Note that the self-checking program should be designed on the basis of extended FMECA [27].

\section{Development tendency of radar PHM}

The development of radar PHM has the following trends:

(1). Towards embedded integration

With the decrement of device volume, weight and power consumption of the microcomputers and the increment of computing power, configuring inside radar units and modules with the so-called intelligent BIT, which integrates distributed data acquisition, processing and centralized fault detection, diagnosis, prediction and warning, becomes the future tendency of PHM. This asks designers and engineers to make full use of the spare resources in high-speed computing logic devices (DSP, CPU and MCU) and programmable ICs, and also to develop corresponding embedded self-checking programs, such that to achieve online and real-time monitoring and analysis.

(2). Concerning together with DFT (Design for Test)

Radar PHM should be based on a well-designed testability. The traditional DFT, when choosing test points and configuring BITs inside an equipment, mainly concerns the detection and isolation of LRU faults, but rarely the fault prediction or PHM. Thus, application of radar PHM needs upgrade of the original system DFT by selecting fault prediction parameters scientifically and configuring the corresponding detection circuits, based on FMECA and analyzing the functional model and failure mechanism of the electric devices.

(3). Remote and distributed PHM

Military radars distribute in broad usually regions geographically and work in bad environment. As a consequence, it is hard to carry out efficient PHM applications with purely the restricted technical force at the local site. Through networking, the computing resources distributed at different radar sites, and the data from working, maintenance and repairing of same-kind radars can be shared for PHM processing, radar design, manufacturing information. And remote experts' knowledge can be collected for the good of fault prediction.

\section{Challenges faced by radar system PHM}

Radar is a complex electronic information system. It contains a large number of sub-systems and modules, which are cross-linked with each other. Its functions, frequency bands and working principles are also different, making the electromagnetic environment inside the radar system extremely complex and difficult to establish physical damage models. A variety of semiconductor components are distributed inside the radar unit 
components. Microscopically, its performance degradation or failure is a series of extremely complicated physical and chemical processes intertwined, which lacks effective theoretical methods for portraying; macroscopically. its faults with many occurrences of suddenness, correlation and uncertainty make it difficult to select the prediction parameters, and the remaining life is difficult to estimate. In engineering applications, a fault may also correspond to multiple predictive parameters. It is difficult to establish a more accurate fault prediction model due to the speed and synchronization limitations of the data collector and the differences in working pressure and environmental conditions under different sampling periods.

In recent years, with the emergence of phased array systems and the continuous development of microelectronics technology, radar unit components have shown new features such as high integration and high speed, together with the reliability, volume and weight of airborne and spaceborne radars. And due to the strict limits of power consumption, the selection of monitoring points and the early warning circuit settings meet difficulties. In addition, the use of FPGA, DSP, ASIC and other logic devices on domestic radars mostly rely on foreign imports, which is limited by "technical blockade", and lack of reliability experimental data for related PHM technology research.

In summary, the implementation of the PHM project of the radar system is still facing many challenges. However, with the large-scale adoption of digital design and manufacturing technology, MEMS, wireless sensor networks and other aspects in the development and production of electronic systems, and the further establishment and improvement of the whole life cycle data and information sharing mechanism, it is not only feasible but also necessary to carry out research on radar system PHM engineering application technology through perfect design and verification evaluation data based on the deep analysis and modeling of the reliability and testability of the radar system.

\section{Conclusion}

With the development of information technology, electronic unit components play an increasingly important role in radar systems. Research on the fault prediction and health management technology, finding potential faults or performance degradation has a great significance on improving the operational safety of radar systems, and reducing the total Life cycle costs

The radar system PHM technology is a further extension of the traditional BIT and condition monitoring functions, adding new features such as fault prediction, health assessment and maintenance decisions. To implement the PHM project of the radar system, the testability of the radar unit components must be optimized and upgraded based on the expansion of FMECA. Embedded integration and test design integration, remote distribution will be the future research hotspot of the radar system PHM technology. Despite the many challenges, but the future of radar PHM is bright.

\section{References}

1. Lü Yongle. Methods of Prediction Model Buildings for Airborne Equipment's Working Performance and Applications[D].Beijing University of Astronautics and Aeronautics, 2009: 1-6.

2. Zuo Xianzhang, Kang Jian, etc. Overview of Fault Prediction Technology[J]. Fire Control \& Command Control, 2010, 35(1): 1-5. 
3. Lian Guangyao, Lv Xiaoming, etc. Study of Key Realization Technology for Fault Prediction System of Electronic Equipment Based on PHM[J]. Computer Measurement \& Control, 2010, 18(9): 1959-1961.

4. Bodden D. S., Hadden W., Grube B. E., etal. PHM as a Design Variable in Air Vehicle Conceptual Design[A]. Aerospace Conference 2005[C]. IEEE, 2005: 1-11.

5. Fraser K. F.. An Overview of Health and Usage Monitoring Systems(HUMS) for Military Helicopters[R]. Australia: Defence Science and Technology Organization, Aeronautical and Martime Reseach Laboratory, 1994.

6. Zhang Baozhen. Development and Application of Prognostics \& Health Management Technology[J]. Measurement \& Control Technology, 2008, 27(2): 5-7.

7. Figueroa F., Holland R., Schmalzel J., etal. Integrated System Health Management (ISHM): Systematic Capability Implementation[A]. Sensors Applications Symposium 2006[C]. IEEE, 2006: 202-206.

8. Mackey R., Iverson D., Pisanich G., etal. Integrated System Health Management (ISHM) Technology Demonstration Project Final Report[R]. California, USA: National Aeronautics and Space Administration, 2006.

9. Michael E. Malley. A Methodology for Simulating The Joint Strike Fighter's Prognostics and Health Management System[D]. Air Force Institute of Technology, 2001.

10. Fang Jiayong, Xiao Mingqing, Huang Hongwei, etc. Fault Prognosis Parameters Selection and Setting in Electronic Equipment[J]. Journal of Air Force Engineering University(Natural Science Edition), 2010, 11(4): 11-15.

11. Han Guotai. Prognostics and Health Management of Avionics[J]. Avionics Technology, 2009, 40(1): 30-38.

12. Nikhil Vichare and Michael Pecht. Prognostics and Health Monitoring of Electronics[J]. IEEE Transactions on Componets and Packaging Technologies, 2006,29(1): 222-229.

13. Chen Shijie, Lian Ke, Wang Houjun. Fault Diagnosis M ethod of Radar ReceiverUsing MultiSignal Flow Graphs Model[J].Journal ofUniversity ofElectronic Science and Technology of China, 2009, 38(1):87-91.

14. Pawlak z, Skowron A. Rough sets: Some extensions . Information Sciences, 2007,177(1): 28-40.

15. Simon Haykin. Neural Networks: A Comprehensive Foundation, second edition[M]. Prentice Hall, 2001: 256-312.

16. Thomas Weise, Raymond Chiong, Ke Tang. Evolutionary Optimization: Pitfalls and Booby Traps[J]. Journal of Computer Science and Technology, 2012, 27(5): 907-936.

17. Li Wenjuan, Ma Cunbao, He Ermin. Framework and Key Technologies of the Integrated Vehicle Health Management System[J]. Advances In Aeronautical Science and Engineering, 2011,2(3):330-334.

18. Chen Ying, $\mathrm{Xu}$ Gang, Gu GuoChang. A Data Mining Approach Based on the Integration of Ontology and Context Knowledge[J], Journal of Software, 2007, 18(10): 2507-2515.

19. Kuo R. J., WANG H. S., et al. Application of Ant K-Means on Clustering Analysis[J]. Computers\& Mathematics, 2005, 50: 1709-1724.

20. Box G. P. E., Jenkins G. M., Reinsel G. C.. Time Series Analysis: Forecasting and Control[M]. 3rd Edition. Pearson Education Asia Ltd., 2005. 
21. Zheng Kai, Hu Jie. A Bayesian Inference Method for Model Extrapolation Together with Qualitative Knowledge[J], Journal of Shanghai Jiaotong University.

22. Zhao Ningshe, Zhai Zhengjun, Wang Guoqing. Technologies of New Generation Avionics Integration and Prognostics and HealthManagement[J]. Measurement \& Control Technology. 2011, 30(1): 1-5, 9.

23. Shi Yibing, Wang Houjun. Key Techniques in Product Design of High-speed Logic Analyzer[J]. Chinese Journal of Scientific Instrument, 2002, z3:38-40.

24. Ramakrishnan, A. etal. A Life Consumption Monitoring Methodology for Electronic Systems[J]. IEEE Transactions on Components and Packaging Technologies, 2003, 26(3): 625-634.

25. Li Chun, Di Mary. Application of Prognostic Technology in IC Design[J]. Semiconductor Technology, 2009, 34(3): 279-282,286.

26. Tan Lin, Guo Chiming, Cheng Zhijun. Optimal maintenance decisions for gamma deteriorating systems[J]. Journal of Systems Engineering and Electronics, 2011, 22(5): 788-793.

27. Shi Junyou, Ji Chao. Study on Enhanced FM ECA M ethod Application[J].Measurement \& Control Technology, 2011, 30(5): 111-114. 